\title{
Photosensitized Killing of Cultured Fibroblasts from Patients with Peroxisomal Disorders Due to Pyrene Fatty Acid-mediated Ultraviolet Damage
}

\author{
G. Hoefler," E. Paschke, ${ }^{\star}$ S. Hoefler, ${ }^{\star}$ A. B. Moser," and H. W. Moser" \\ Departments of ${ }^{*}$ Medical Biochemistry and ${ }^{\ddagger}$ Pediatrics, Karl Franzens University, Graz, Austria; and ${ }^{\S}$ Kennedy Institute, \\ Johns Hopkins School of Medicine, Baltimore, Maryland 21205
}

\begin{abstract}
The influence of pyrene-fatty acids on the resistance of cells to ultraviolet (UV) radiation was investigated in cultured fibroblasts from patients with five types of peroxisomal disorders. All showed reduced survival compared to control. The effect varied with the biochemical defect involved and the chain length of the pyrene fatty acid. Reduced survival was observed in cells deficient in plasmalogens (rhizomelic chondrodysplasia punctata) and in cells deficient in peroxisomal fatty acid oxidation (bifunctional enzyme deficiency), which accumulated pyrene-fatty acids. X-linked adrenoleukodystrophy fibroblasts accumulated pyrene-fatty acids and showed increased UV sensitivity only when exposed to longer-chain pyrene fatty acids. UV radiation resistance was lowest in cells with combined impairment of plasmalogen synthesis and fatty acid oxidation (Zellweger syndrome, neonatal adrenoleukodystrophy), suggesting that UV sensitivity correlates inversely with the ratio of plasmalogens to radical producing substances.

Fibroblasts deficient in plasmalogens gained normal UV resistance when their plasmalogen levels were normalized by hexadecylglycerol. UV resistance increased when Zellweger cells were fused with $X$-linked adrenoleukodystrophy cells, and also when Zellweger cells belonging to different complementation groups were fused.

The results provide leads to the pathogenesis of the multiple malformations associated with peroxisomal disorders and a method for the selection of cells in which the metabolic defect has been corrected. (J. Clin. Invest. 1991. 88:1873-1879.) Key words: radicals $\bullet$ lipids $\bullet \beta$-oxidation $\bullet$ plasmalogens $\bullet$ genetic complementation
\end{abstract}

\section{Introduction}

The peroxisomal disorders Zellweger syndrome (ZS) ${ }^{1}$ and rhizomelic chondrodysplasia punctata (RCDP) are associated with malformations of multiple organs and lead to early death.

Address reprint requests to Dr. Hoefler, Institute of Pathology, University of Graz, Auenbruggerplatz 25, A-8036 Graz, Austria.

Received for publication 22 January 1991 and in revised form 19 June 1991

1. Abbreviations used in this paper: $\beta$-ox-def, a female patient with an unidentified defect of peroxisomal $\beta$-oxidation; HDG, hexadecylglycerol; NALD, neonatal adrenoleukodystrophy; PFA, pyrene fatty acid; PFA-MEM, minimal essential medium, supplemented with pyrene fatty acids; P6, 6-(1'-pyrene)hexanoic acid; P10, 10-(1'-pyrene)decanoic acid; P12, 12-(1'-pyrene)dodecanoic acid; P16, 16-(1'-pyrene)hexa-

J. Clin. Invest.

(c) The American Society for Clinical Investigation, Inc.

$0021-9738 / 91 / 12 / 1873 / 07 \quad \$ 2.00$

Volume 88, December 1991, 1873-1879
Although ZS was formerly assigned to the "multiple congenital anomaly" disease category (1), it is now generally accepted that the basic abnormality is the failure to form normal peroxisomes (2). Multiple peroxisomal functions are defective, including the formation of plasmalogens and certain bile acids, and the oxidation of very long chain fatty acids (VLCFA), phytanic acid, and pipecolic acid. RCDP has also been shown to be a peroxisomal disorder $(3,4)$, but the defect is more restricted since the organelle is formed, and peroxisomal malfunctions are confined to defective synthesis of plasmalogens, phytanic acid oxidation, and the processing of one of the peroxisomal enzymes, 3-oxoacyl-coenzyme A thiolase.

There is as yet no information about the mechanisms by which peroxisomal malfunction leads to the profound disturbances associated with ZS and RCDP. The accumulations of VLCFA and bile acid intermediates probably play a role since multiple malformations, including those of the brain, are common to ZS and certain peroxisomal disorders in which biochemical defects are confined to VLCFA and bile acid metabolism $(5,6)$. Instead it is the defects of plasmalogen synthesis and phytanic acid oxidation that represent a point of commonality between ZS and RCDP.

An important potential pathogenetic mechanism was suggested by a recent report of Zoeller et al. (7) in their studies of Chinese hamster ovary cells that lack peroxisomes and demonstrate the broad array of biochemical defects characteristic of ZS (8). When those cells were cultivated in the presence of the photosensitizer 12-(1'-pyrene)dodecanoic acid (P12), they were more sensitive to ultraviolet (UV) irradiation than normal cells. This hypersensitivity has been attributed to the lack of protection against singlet oxygen or radicals produced by P12. Singlet oxygen and radicals can cause extensive damage to lipids, proteins, and nucleic acids (9). Such damage could be a plausible explanation for the multiple malformations associated with human peroxisomal disorders. In this article we demonstrate that cell lines from patients with peroxisomal disorders also show increased sensitivity to pyrene fatty acid (PFA)-mediated UV damage. By comparing this sensitivity in human mutants with different biochemical lesions, such as ZS, RCDP, and isolated defects of peroxisomal $\beta$-oxidation of VLCFAs, we were able to focus specifically on the role of plasmalogen deficiency.

\section{Methods}

Materials and general methods. 6-(1'-Pyrene)hexanoic acid (P6), 10(1'-pyrene)decanoic acid (P10), and P12 were purchased from Lambda Probes, Graz, Austria. 16-(1'-Pyrene)hexadecanoic acid (P16) was obtained from Molecular Probes Inc., Junction City, OR. 1-O-hexadecyl$s n$-glycerol was kindly provided by F. Paltauf, Technical University of

decanoic acid; RCDP, rhizomelic chondrodysplasia punctata; UV, ultraviolet; VLCFA, very long chain fatty acid; X-ALD, X-linked adrenoleukodystrophy; ZS, Zellweger syndrome. 
Graz. All other chemicals were of analytical grade and obtained from commercial sources.

Cells and culture conditions. Fibroblasts were obtained for diagnostic purposes at the Kennedy Institute. Diagnosis was based on the following biochemical criteria: VLCFAs (10) and phytanic acid (11) in serum, oxidation of VLCFAs in fibroblasts (4), plasmalogen biosynthesis in cultured fibroblasts (12), accumulation of bile acid intermediates (13) and pipecolic acid (14) in serum, subcellular distribution of catalase (15), and, in some cases, immunoblot analysis of peroxisomal $\beta$ oxidation enzymes in liver homogenates and fibroblasts (6). The patients with ZS and neonatal adrenoleukodystrophy (NALD) belong to complementation groups 1, 2, and 3 described in Roscher et al. (16). The patient diagnosed as RCDP is case 2 in Hoefler et al. (4). A patient with a deficiency of peroxisomal bifunctional enzyme was reported by Watkins et al. (6), and a female patient with an unidentified defect of VLCFA oxidation ( $\beta$-ox-def) by Naidu et al. (17).

All cell lines were cultured at $37^{\circ} \mathrm{C}$, in a $5 \% \mathrm{CO}_{2}, 95 \%$ air atmosphere in minimal essential medium (MEM) with Earle's salts supplemented with fetal bovine serum ( $10 \% \mathrm{vol} / \mathrm{vol})$, glutamine ( $1 \mathrm{mM})$, penicillin G (10,000 IU/liter), and streptomycin (0.1 g/liter). PFA were prepared as a $10 \mathrm{mM}$ stock solution in dimethyl sulfoxide and mixed into MEM to obtain the desired concentrations (PFA-MEM).

UV irradiation. Approximately $2 \times 10^{5}$ cells were seeded into 10$\mathrm{cm}^{2}$ dishes, cultured overnight in MEM, washed with PBS, and incubated with PFA-MEM for $24 \mathrm{~h}$. Immediately before UV irradiation, cells were washed twice with PBS and incubated with fresh, PFA-free MEM. Culture dishes were placed on a 1-mm-thick glass plate at a distance of $5 \mathrm{~cm}$ above a transilluminator (Macrovue model 2011, LKB Produkter, Bromma, Sweden) and exposed to UV light for $5 \mathrm{~min}$. The intensity was $0.17 \mathrm{~mW} / \mathrm{cm}^{2}$ at $313 \mathrm{~nm}$, when measured through the glass plate with an International Light Inc. (Newburyport, MA) model 1700 (detector SED 400, interference filter NS 313, and diffuser W 5361). Instead of "vitality testing" by trypan blue exclusion, which gave poorly reproducible results, the ability of cells to attach to the culture dishes was used as a criterion for cell viability.

For that purpose, cells were trypsinized and replated $24 \mathrm{~h}$ after UV treatment. After additional $24 \mathrm{~h}$, the cultures were washed three times with PBS to remove unattached cells. The remaining cells were collected after trypsin treatment and counted in a Fuchs-Rosenthal chamber.

Fluorescence microscopy. Approximately $2 \times 10^{5}$ cells were seeded into $10-\mathrm{cm}^{2}$ dishes containing sterile glass coverslips, incubated with MEM containing $10 \mu \mathrm{M}$ P12, and incubated for $24 \mathrm{~h}$. The coverslips were washed once with MEM, twice with PBS containing $1 \mathrm{mg} / \mathrm{ml}$ bovine serum albumin, and three times with PBS and mounted with Mowiol (Frankfurt am Main, FRG). Photographs were taken with a Zeiss Photomikroskop III (Carl Zeiss, Oberkochen, FRG) with epiillumination with a maximum at $365 \mathrm{~nm}$ (filter combination G 365, FT 395, LP 420) using Kodak TriX Pan (Eastman Kodak Co., Rochester, NY) film. Detection of mitochondria and Golgi apparatus was accomplished using mouse anti-human subcellular particles monoclonal antibodies (Chemicon International, Inc., Temecula, CA). Fibroblasts were processed for immunofluorescence as recommended by the supplier of the antibodies.

Incorporation of PFAs into fibroblasts. Approximately $2 \times 10^{5}$ cells were seeded into $10-\mathrm{cm}^{2}$ dishes and incubated with the indicated concentrations of PFAs for $24 \mathrm{~h}$. They were washed once with MEM, twice with PBS containing $1 \mathrm{mg} / \mathrm{ml}$ bovine serum albumin and three times with PBS, scraped with a rubber policeman in $0.5 \mathrm{ml}$ PBS, and sonicated for $30 \mathrm{~s}$ at $50 \mathrm{~W} .0 .2 \mathrm{ml}$ was added to $0.85 \mathrm{ml}$ of a chloroform/ methanol mixture $(1 / 2, \mathrm{vol} / \mathrm{vol})$ to form a single-phase Bligh and Dyer mixture (18), and the fluorescence was determined using a model $F$ 4000 fluorimeter (Hitachi, Tokyo) at an excitation wavelength of 340 $\mathrm{nm}$ and an emission wavelength of $377 \mathrm{~nm}$. Remaining portions were used for protein determination (19).

Lipid analysis. Approximately $2 \times 10^{6}$ cells were incubated with media supplemented with $10 \mu \mathrm{M}$ P12 for $16 \mathrm{~h}$, washed, and harvested as described above. After Folch extraction the lower phase was evapo- rated to dryness, spotted on plates of silica gel G60, and developed in petrol ether/diethyl ether/acetic acid (30:5:1, vol/vol/vol). Fluorescence of plates was scanned on a scanner (model 400, Elscript, Hirschmann, Munich, FRG).

Subcellular fractionation of fibroblasts. Approximately $2 \times 10^{6}$ cells were incubated with media supplemented with $10 \mu \mathrm{M} P 12$ for $16 \mathrm{~h}$, washed as described above, and harvested by trypsinization. They were homogenized in a ball-bearing homogenizer and fractionated on a linear gradient of increasing (from $15 \%$ to $40 \%$ ) Nycodenz (Sigma Chemical Co., St. Louis, MO) and decreasing (from 0.25 to $0 \mathrm{M}$ ) sucrose concentration essentially as described (6), except that a VTI.80 rotor (Beckman Instruments, Inc., Palo Alto, CA) was used and 15 fractions of $330 \mu \mathrm{l}$ each were collected. $100 \mu \mathrm{l}$ each was used for determination of fluorescence as described above.

Gel filtration on Sepharose 4B. Fractions obtained from Nycodenz gradients were loaded onto a column $(0.5 \times 5 \mathrm{~cm})$ of Sepharose $4 B$ (Pharmacia, Uppsala, Sweden) equilibrated with $0.25 \mathrm{M}$ sucrose containing $10 \mathrm{mM}$ Tris/HCl, pH 7.5, $1 \mathrm{mM}$ EDTA. Fractions of $100 \mu \mathrm{l}$ were collected and the fluorescence was measured as described above.

Hexadecylglycerol (HDG) supplementation. To increase the plasmalogen content in fibroblasts deficient in peroxisomal plasmalogen biosynthesis, cells were incubated with $60 \mu \mathrm{M}$ HDG for $24 \mathrm{~h}$ according to Hermetter et al. (20) before P12 incubation and UV irradiation.

Cell fusion. Parental cell lines were seeded in a $25-\mathrm{cm}^{2}$ flask in a $1: 1$ ratio and fused as reported previously (13), except that the separation of fusion products from unfused cells was omitted. $4 \mathrm{~d}$ after fusion cells were divided into six 10- $\mathrm{cm}^{2}$ dishes and incubated in MEM for $24 \mathrm{~h}$. Cells were washed twice with PBS and cultivated for $24 \mathrm{~h}$ in MEM containing P16 at the concentrations indicated and subjected to the standard UV irradiation protocol. For determination of the percentage of multikaryons, cells were stained with Pappenheim and the number of nuclei was counted in at least 100 cells.

\section{Results}

Increased sensitivity of fibroblasts from patients with peroxisomal disorders to P12-UV treatment. Fibroblasts from patients with ZS, NALD, X-linked adrenoleukodystrophy (X-ALD), RCDP, bifunctional enzyme deficiency, and $\beta$-ox-def were incubated in MEM supplemented with P12 at concentrations from 2.5 to $12.5 \mu \mathrm{M}$ for $24 \mathrm{~h}$. After replacement with normal MEM cells were UV-irradiated for $5 \mathrm{~min}$ and the number of surviving cells was determined (Fig. $1 \mathrm{c}$ ). At concentrations of 5 $\mu \mathrm{M}<5 \%$ of fibroblasts from patients with ZS, NALD, and bifunctional enzyme deficiency survived, whereas $60 \%$ of control and X-ALD cells were still viable. Cells from a patient with RCDP were slightly less affected but clearly more sensitive than controls. At a concentration of $2.5 \mu \mathrm{M}$ only cells from ZS were affected, but not other cell lines. Concentrations up to $20 \mu \mathrm{M}$ without UV irradiation did not affect cell viability. UV irradiation alone decreased the number of surviving cells by about $10 \%$ (data not shown).

Increased sensitivity of $X-A L D$ fibroblasts to P16-UV treatment. Fibroblasts from patients with ZS, NALD, X-ALD, RCDP, and $\beta$-ox-def were incubated in MEM supplemented with P16 at concentrations ranging from 5 to $15 \mu \mathrm{M}$ for $24 \mathrm{~h}$ and subjected to the standard UV irradiation protocol (Fig. 1 $d$ ). In contrast to the results with $\mathrm{P} 12$, cells from patients with X-ALD and $\beta$-ox-def showed increased sensitivity to this treatment. At 7.5 and $12.5 \mu \mathrm{M}<10 \%$ of the cells were viable as compared to $55 \%$ and $35 \%$ of controls.

To demonstrate the reproducibility of this method and to account for possible variations among different cell lines and passage numbers, fibroblasts from three patients with X-ALD and three controls were measured in duplicate and mean and 

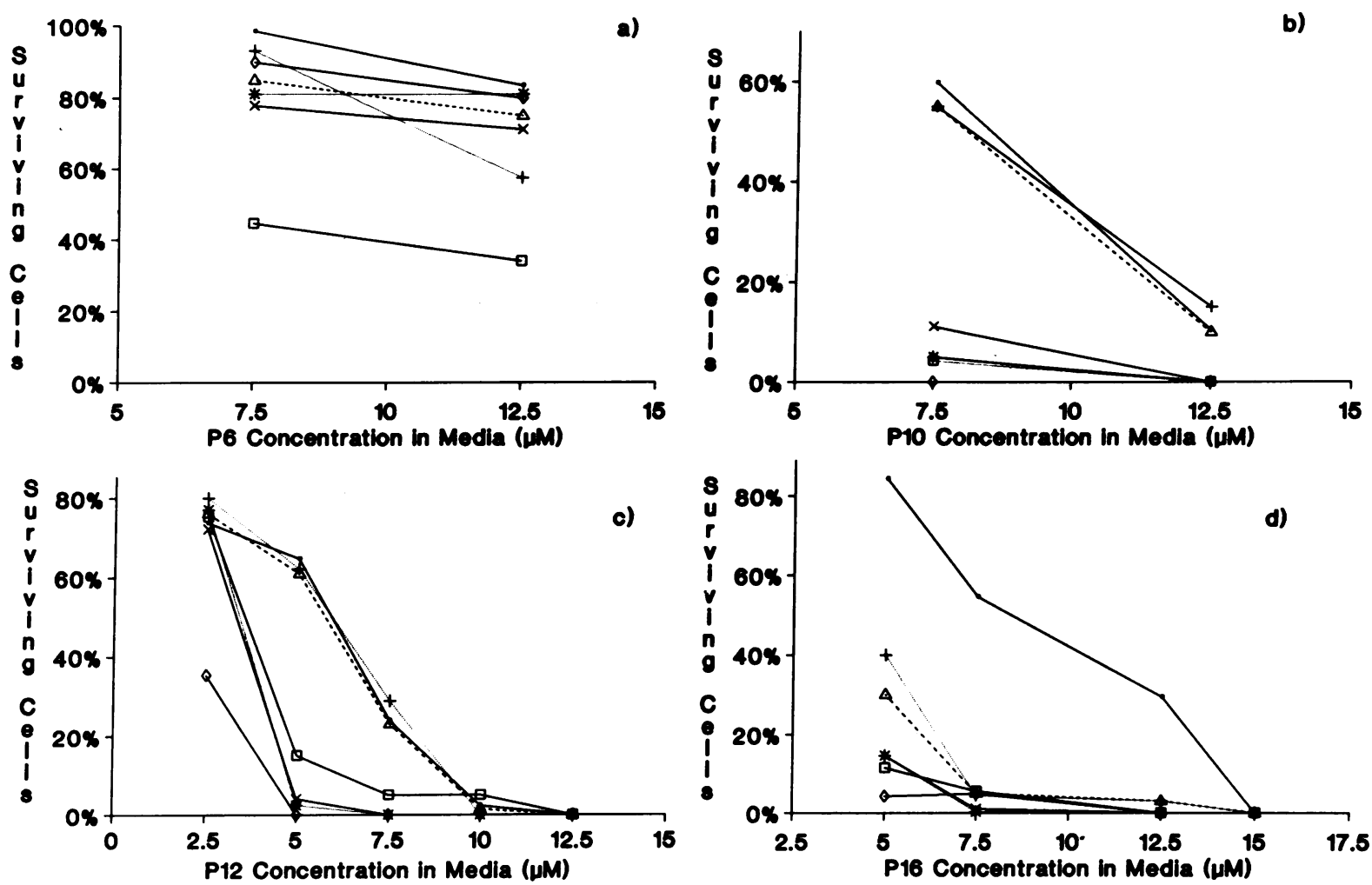

Figure 1. Percentage of fibroblasts surviving PFA-UV treatment. Cells were incubated with MEM containing (a) P6, (b) P10, (c) P12, and (d) P16 PFAs at the concentrations indicated and subjected to UV irradiation as described in Methods. The ability of fibroblasts to attach to culture dishes after trypsinization was taken as a criterion for cell viability. (•) Control, $(+)$ X-ALD, (*) NALD, (ם) RCDP, ( $\times$ ) bifunctional enzyme deficiency, $(\diamond) \mathrm{ZS},(\Delta) \beta$-ox-def.

standard deviation were determined (Fig. 2). At concentrations of 7.5 and $12.5 \mu \mathrm{M}$ a clear difference between control and disease cells was observed.

Chain-length specificity of peroxisomal oxidation of PFA. In order to determine the chain length of PFAs that require peroxisomal $\beta$-oxidation, fibroblasts from patients with ZS, bifunctional enzyme deficiency, and RCDP and controls were incubated in media containing 7.5 and $12.5 \mu \mathrm{M}$ of P6 (Fig. $1 a$ ) and P10 (Fig. $1 b$ ). For P10 essentially the same results were obtained as for P12 and P16. After P6 incubation however, only RCDP cells seemed to be sensitive to the UV treatment. Only $45 \%$ and $35 \%$ of the cells survived as compared to $>70 \%$ and $60 \%$ of all other cells.

Accumulation of PFAs in fibroblasts from patients with defective peroxisomal $\beta$-oxidation. Intracellular content of P1 2 in cells from a patient with ZS and a control was compared using fluorescence microscopy (Fig. 3). After a 24-h incubation with P12, fibroblasts showed an accumulation of fluorescent material in a distinct punctate pattern. It was more pronounced in ZS and did not match to the staining pattern obtained with antibodies against mitochondria or Golgi apparatus.

To quantify the incorporation of P6, P10, P12, and P16 into fibroblasts from patients with ZS, NALD, X-ALD, RCDP, and $\beta$-ox-def and controls, fluorescence was measured after incubation with PFAs for $24 \mathrm{~h}$ at concentrations indicated (Fig. 4). Virtually no differences in the incorporation of P6 into cells could be found (data not shown). Cells from patients with ZS and NALD accumulated two to five times more P10 (Fig. $4 a$ ) and P1 2 (Fig. $4 b$ ) than controls, X-ALD, RCDP, and $\beta$-ox-def.
For P16 (Fig. $4 c$ ) the intracellular accumulation was less pronounced than for P10 and P12 (50-150\% over controls). In contrast to P10 and P12 however, P16 was stored in fibroblasts from patients with X-ALD and $\beta$-ox-def. Intracellular concentrations of all PFAs in RCDP fibroblasts were comparable to controls.

Incorporation of $\mathrm{P} 12$ into different lipid classes were similar in ZS, bifunctional enzyme deficiency, and control (Table I)

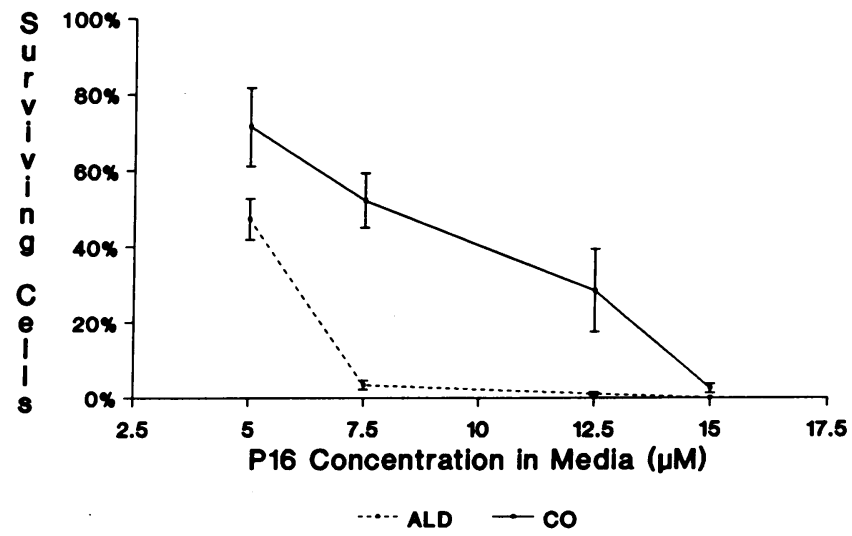

Figure 2. Reproducibility of UV sensitivity testing. Fibroblasts from three patients with X-ALD and three controls were incubated with P16-MEM at the concentrations indicated and subjected to the standard UV irradiation protocol in duplicate. Percentage of surviving fibroblasts is given as mean \pm standard deviation $(n=6)$. 

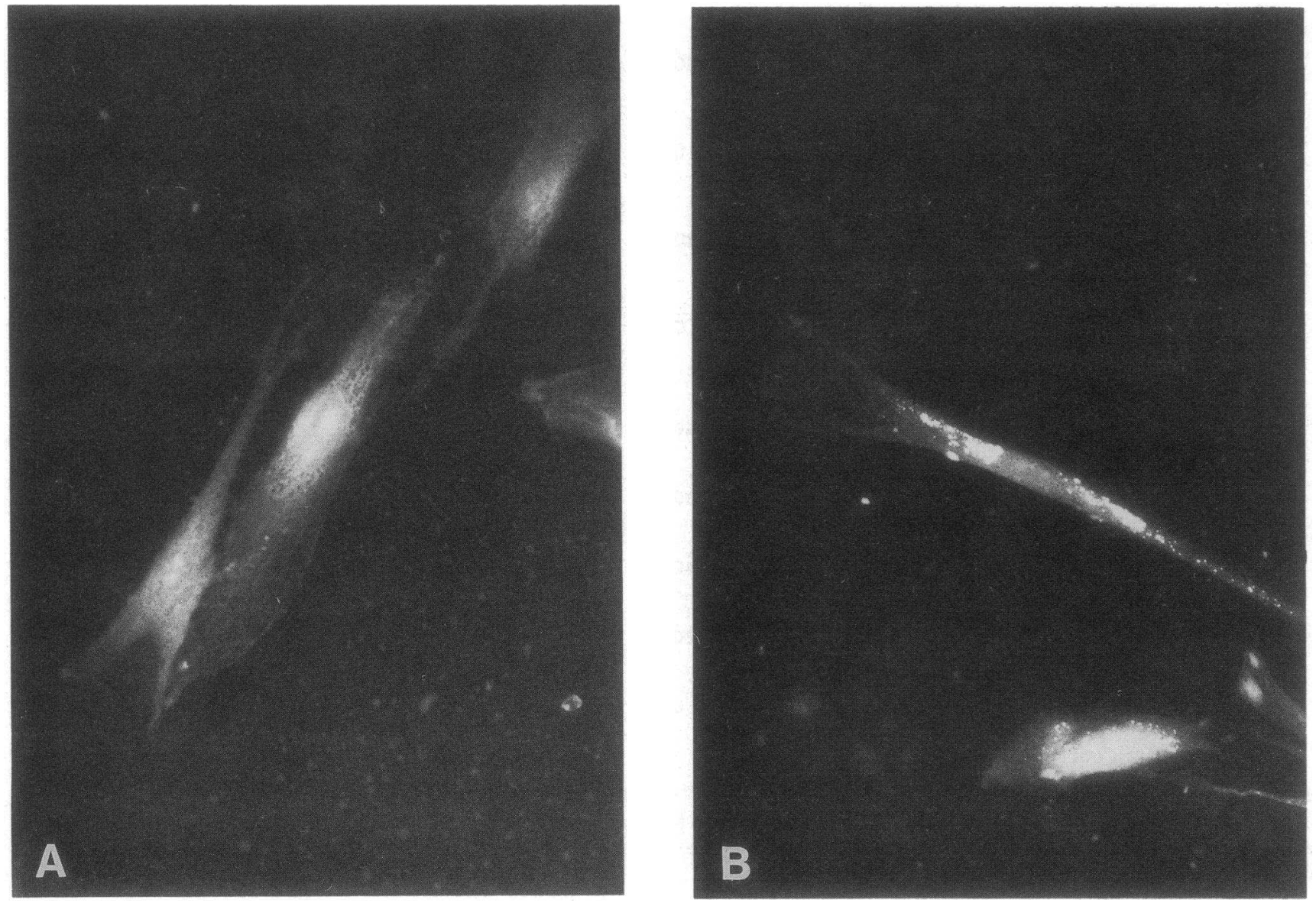

Figure 3. Fluorescence microscopy of PFA-treated fibroblasts. Cells were grown on coverslips and incubated with $10 \mu \mathrm{M}$ P12 for 24 h. After extensive washing with PBS containing bovine serum albumin $(1 \mathrm{mg} / \mathrm{ml})$ and PBS, the cells were mounted and viewed as described in Methods. $(A)$ Control fibroblasts, $(B)$ fibroblasts from a patient with ZS. $\times 528$.

with the exception that the percentage of unesterified PFAs was slightly higher.in the $\beta$-oxidation-deficient fibroblasts.

Subcellular localization of PFA incorporated into fibroblasts. To investigate the subcellular distribution of PFA, fibroblasts from control and a patient with ZS were incubated with $10 \mu \mathrm{M}$ P12 for $16 \mathrm{~h}$. After extensive washes they were homogenized and fractionated on a continuous Nycodenz gradient. $49 \%$ (control) and $48 \%$ (ZS) of the fluorescence label was localized in the two fractions with least density (cytosolic fractions). The remaining portion was distributed evenly over the gradient and no association with other marker enzymes was found.

The two topmost fractions were pooled and analyzed on a Sepharose 4B gel filtration column, which has an exclusion limit of $2 \times 10^{7} \mathrm{~mol} w \mathrm{wt}$ for globular proteins. $82 \%$ of the fluorescence label was recovered in the void volume.

Supplementation with HDG. Peroxisomal steps of plasmalogen biosynthesis can be bypassed by feeding cells with HDG (20). Accordingly, the plasmalogens can be raised to near normal levels in RCDP and ZS fibroblasts despite their peroxisomal dysfunction of plasmalogen biosynthesis. Thus we were able to discriminate between the effects of an increased exposure to free radicals after UV irradiation in the presence of PFAs and the possible function of plasmalogens in protecting them against these noxes. Cultures were supplemented with HDG and subjected to UV irradiation after incubation with P12 (Fig. 5). Fibroblasts from patients with RCDP gained a resistance comparable to controls whereas there was no change in the $\beta$-oxidation-deficient cell mutants, ZS and X-ALD. Similarly HDG did not affect the resistance of control fibroblasts to the P12-UV treatment (data not shown).

Normal resistance of heterokaryotic $X-A L D / Z S$ cells to $P F A-U V$ treatment. Among cell lines from patients with disorders of peroxisome biogenesis, five different complementation groups, which were characterized by a normalization of plasmalogen biosynthesis in multikaryotic cells upon successful genetic complementation, could be described by our group (16).

In order to test the possibility that PFA-UV treatment could provide a method of selecting for biochemically normal cells in $\beta$-oxidation or plasmalogen-deficient mutants, we fused cell lines from different complementation groups. The resulting mixture of monokaryotic and multikaryotic cells was subjected to PFA-UV treatment. For example, without receiving the treatment $\sim 10 \%$ of cells had two or more nuclei after fusion of fibroblasts from patients ZS1 (complementation group 1) and ZS2 (complementation group 2). With increasing concentrations of $\mathrm{P} 12$, the proportion of multikaryotic cells rose to $>50 \%$ after UV irradiation (Fig. 6). The increased resistance of multikaryotic cells to PFA-UV treatment was also observed when ZS1 fibroblasts or ZS2 fibroblasts were fused with fibroblasts from a patient belonging to complementation group 3: $55 \%$ and $41 \%$ multinuclear cells were observed after PFA- 

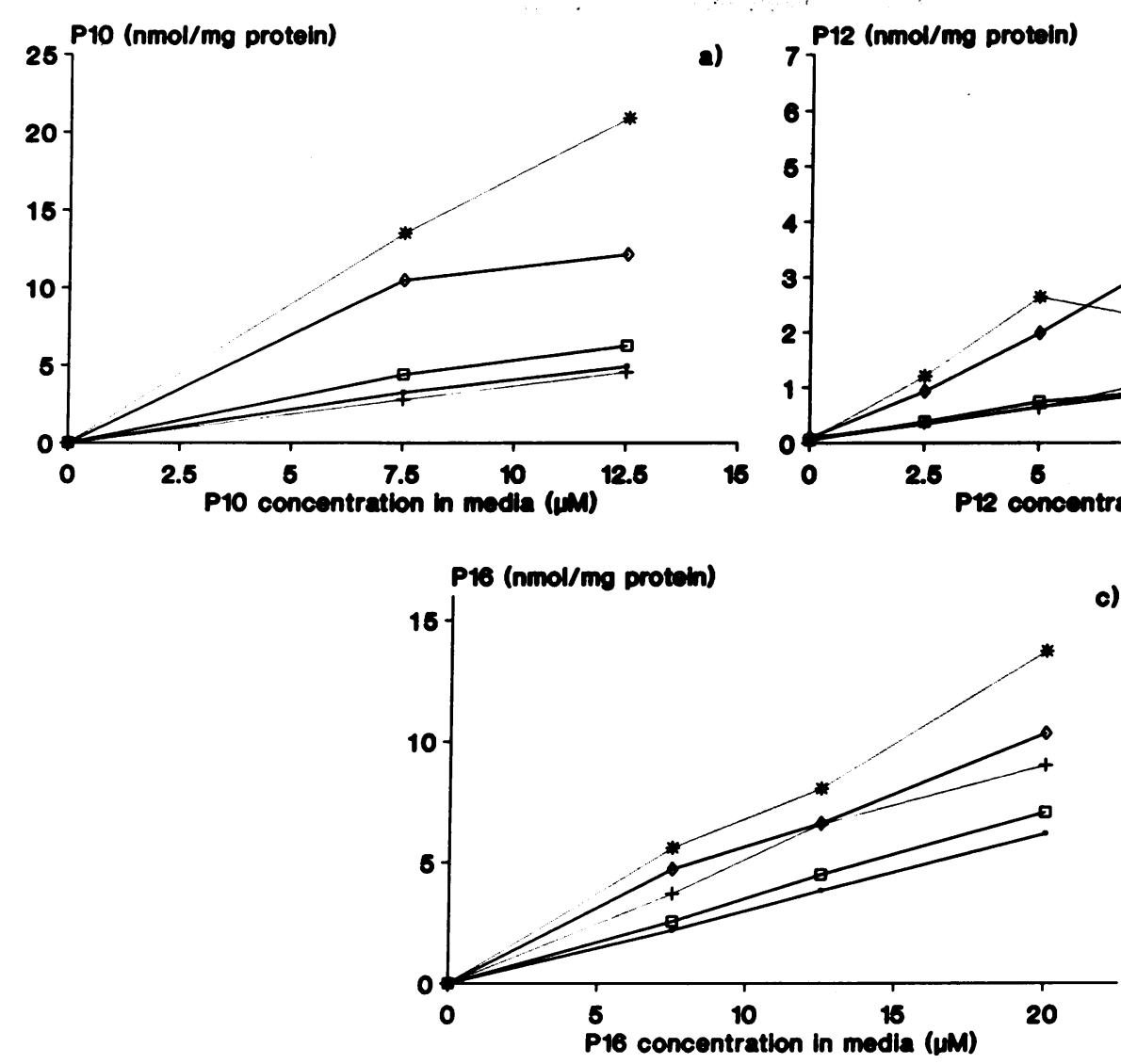

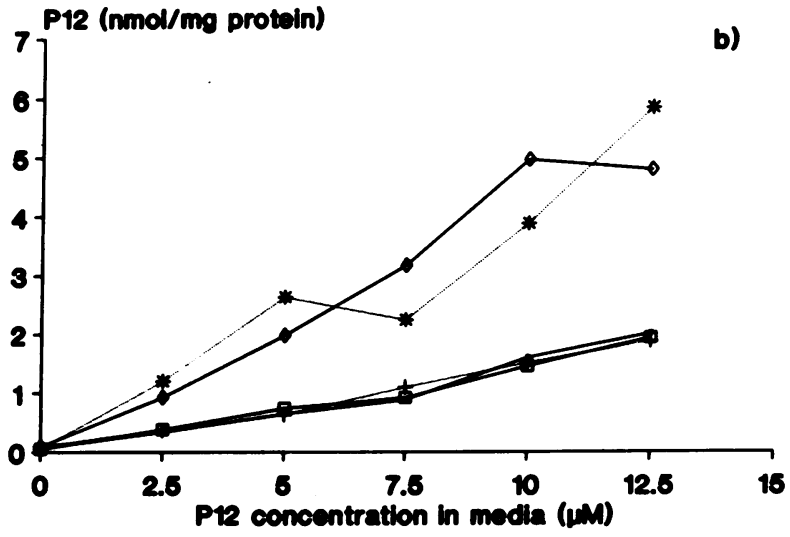

c)

Figure 4. Incorporation of PFAs into fibroblasts from patients with peroxisomal disorders. Cells were incubated with MEM containing (a) P10, (b) P12, and (c) P16 PFAs at the concentrations indicated for $24 \mathrm{~h}$. The fluorescence was determined after extraction with a single-phase Bligh

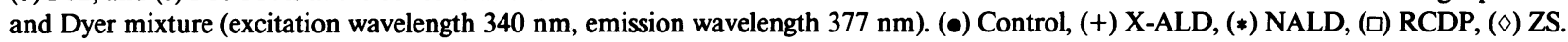

UV treatment with $15 \mu \mathrm{M}$ P12 as compared to $7 \%$ and $5 \%$ without treatment. We conclude that the defect causing higher susceptibility to the PFA-UV treatment had been corrected in fused cells from patients with disorders of peroxisome biogenesis belonging to different complementation groups.

Similar results were obtained when cells from a patient with X-ALD were fused with those of patient ZS1 (complementation group 1). The number of multikaryotic cells increased from $20 \%$ to over $50 \%$ after PFA treatment (Fig. 6). This indicates that different genetic defects cause the increased sensitivity of X-ALD and ZS fibroblasts to PFA-induced UV damage. Fusing X-ALD cells with fibroblasts from complementation

Table I. P12 Incorporated into Lipids Isolated from Cultured Fibroblasts

\begin{tabular}{lccr}
\hline & Control & ZS & BED \\
\hline & & $\%$ & \\
Phospholipids & 63.5 & 54.2 & 55.9 \\
Triglycerides & 13.8 & 11.8 & 14.4 \\
Cholesterol esters & 7.2 & 10.8 & 9.7 \\
Unincorporated PFA & 15.5 & 23.2 & 20.1 \\
\hline
\end{tabular}

After incubation of fibroblasts in MEM containing $20 \mu \mathrm{M}$ P12, separation and detection were performed as described in the Methods section. Abbreviation: BED, bifunctional enzyme deficiency. group 2 or 3 gave analogous results. The percentage of multikaryotic cells was more than threefold higher in PFA-UV treated compared to untreated cells. No increase was observed when either control cells or diseased cells were fused among themselves (Fig. 6). This indicates that multikaryotic cells generally are not more resistant than monokaryotic cells.

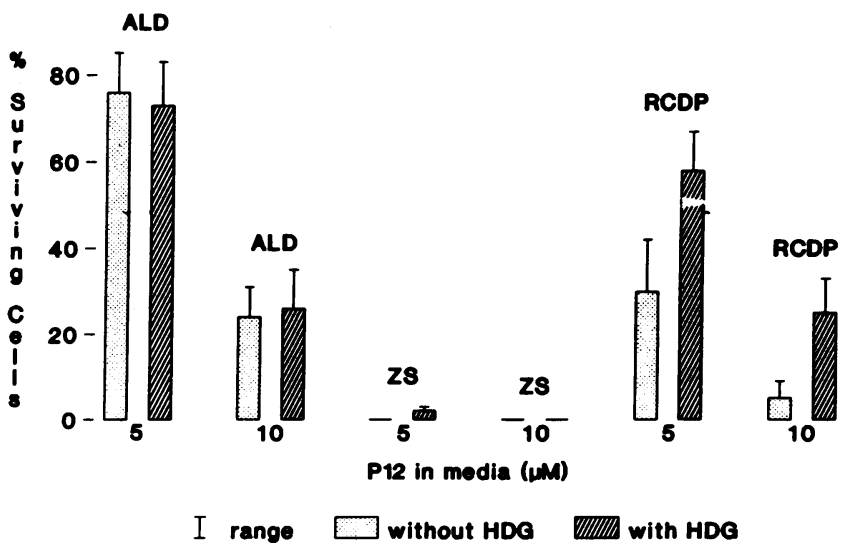

Figure 5. UV resistance of HDG-substituted fibroblasts. Cells from patients with X-ALD $(n=2), \operatorname{ZS}(n=3)$, and $\operatorname{RCDP}(n=3)$ were incubated with $60 \mu \mathrm{M}$ HDG for $24 \mathrm{~h}$ before P12 incubation. After UV irradiation cell viability was determined as described in Methods. All experiments were done in duplicates, the range of all results is given. 


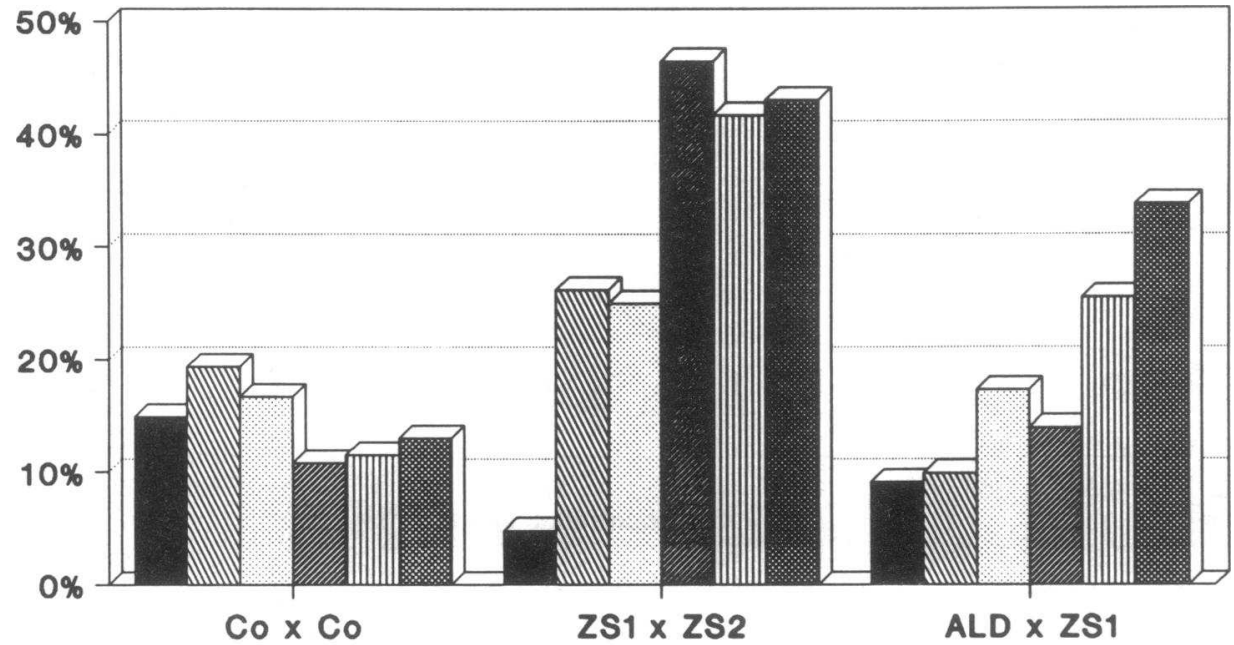

$0 \mu \mathrm{M}$

$12.5 \mu \mathrm{M}$

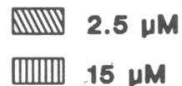

$15 \mu \mathrm{M}$
$10 \mu \mathrm{M}$

$20 \mu \mathrm{M}$

P16 Concentration in Media

Figure 6. Complementation analysis of fibroblasts from patients with peroxisomal disorders. Cells were fused using polyethylene glycol and subjected to UV irradiation after incubation with P16 at the concentrations indicated. Among surviving cells, the percentage of multinuclear cells was determined after staining with Pappenheim. An increase indicates correction of the defect causing the higher susceptibility of monokaryotic cells.

\section{Discussion}

Fibroblasts from patients with disorders of peroxisome biogenesis exhibit decreased resistance to UV irradiation after incubation with PFAs of chain lengths between $\mathrm{C} 10$ and $\mathrm{C} 16$. This phenomenon can be explained by two facts: First, P12-CoA has been shown to be oxidized in peroxisomes (21) and PFAs accumulate in cells deficient in peroxisomal $\beta$-oxidation. Second, plasmalogens are markedly reduced in these cells.

Zoeller et al. (7) presented evidence that the deficiency of plasmalogens is responsible for the increased sensitivity of Chinese hamster ovary cell mutants with defective peroxisome biogenesis which exhibit combined defects of plasmalogen synthesis and $\beta$-oxidation. Supplementation of these cells with HDG, thereby increasing the plasmalogen content, restored resistance to PFA-UV treatment.

Our intention was to separate these two possible mechanisms using fibroblast mutants defective in plasmalogen biosynthesis only (RCDP) and defective peroxisomal $\beta$-oxidation only (bifunctional enzyme deficiency). In both cases mutant cells were more strongly affected by PFA-UV treatment than controls (Fig. 1). Cells with combined deficiencies (ZS) appeared to be influenced at even lower concentrations of P12. We postulate therefore that the ratio of plasmalogens to radical-producing substances (PFAs) is responsible for rendering cells with peroxisomal defects more susceptible to UV-induced cell damage.

This suggestion is supported by close examination of our data on the intracellular content of PFAs and the sensitivity of cells to UV irradiation (Fig. 1) and the intracellular content of PFAs (Fig. 4): at a concentration of $10 \mu \mathrm{M}$ P12 in media, the intracellular amount of $\mathrm{P} 12$ was $1.59 \mathrm{nmol} / \mathrm{mg}$ protein in control fibroblasts, resulting in survival of $3 \%$ after UV irradiation. In $\beta$-oxidation-deficient cells a similar intracellular content $(1.97 \mathrm{nmol} / \mathrm{mg}$ protein) and survival rate $(4 \%)$ was already obtained at $5 \mu \mathrm{M}$. Concentrations of $<1 \mathrm{nmol} / \mathrm{mg}$ protein affected plasmalogen-deficient fibroblasts only. Furthermore, supplementation with HDG protected only plasmalogen-deficient, but not $\beta$-oxidation-deficient cells (Fig. 5).

Incorporation of P12 into different lipid classes in $\beta$-oxidation-deficient cells (ZS and $\beta$-ox-def) was similar to control (Table 1). In subcellular fractionation experiments $\sim 50 \%$ of the fluorescent material was found in particles with a density lower than subcellular organelles. We speculate that these might represent the particles observed in fluorescence microscopy, e.g., lipid droplets.

Although an elevation and defective oxidation of VLCFAs is characteristic for fibroblasts from patients with X-ALD, their sensitivity against UV irradiation and the incorporation of PFAs after incubation with P10 or P12 was equal to control cells. With P16 however, the PFA incorporation was higher than in controls and the cell damage after UV treatment was comparable to other cell lines with peroxisomal diseases. A defective peroxisomal VLCFA ligase has been proposed to be the cause of X-ALD $(22,23)$. Possibly this VLCFA ligase could be specific for PFAs with a chain length higher than P12. Generally, higher concentrations of P16 in media were required to obtain the same effects than with $\mathrm{P} 10$ or P12. This might either be explained by a poorer solubility and hence a lower bioavailability of P16 or by a lower rate of activation by VLCFA ligase.

Six complementation groups of disorders of peroxisome biogenesis have so far been established by cell fusion experiments $(15,24)$. The restoration of plasmalogen biosynthesis and the ability to form peroxisomes as evidenced by the formation of particle-bound catalase were used as criteria for successful genetic complementation. Peroxisomal $\beta$-oxidation has been measured in fused fibroblasts and complementation experiments using cells from patients with isolated defects in peroxisomal $\beta$-oxidation have been performed (25). No data exist on whether this pathway is restored in fused cells from patients with ZS and X-ALD.

Our results (Fig. 6) show that fused cells from patients with ZS belonging to different complementation groups (16) gain resistance to PFA-UV treatment. This indicates, that in addi- 
tion to plasmalogen synthesis also the ability to metabolize PFAs is restored. When fibroblasts from patients with ZS belonging to different complementation groups were fused with $\mathrm{X}$-ALD fibroblasts, an increase of UV resistance was observed. This can be taken as evidence that the genetic defects causing the deficiencies of peroxisomal $\beta$-oxidation in ZS and X-ALD are different.

In general, the PFA-UV treatment could be a useful screening test for peroxisomal disorders. Although there are many established methods (2) such as measuring levels or oxidation of VLCFAs, determination of plasmalogen synthesis or dihydroxyacetonephosphate-acyl transferase activity, this test has some advantages. Fibroblasts are more sensitive to PFA-UV treatment when they are deficient either in peroxisomal $\beta$-oxidation or plasmalogen synthesis alone or in a combination of both. The equipment necessary for this test is minimal and a simple UV source can be used after calibration. The incorporation of PFAs can be measured in order to differentiate between defects of $\beta$-oxidation and plasmalogen synthesis.

Another potential application of the method might be the selection of corrected cells in reverse genetics. Fibroblasts with defects in plasmalogen synthesis and/or peroxisomal $\beta$-oxidation could be transfected with a cDNA library, subjected to PFA-UV treatment, and used for isolation of gene(s) responsible for the defect. A similar procedure has been successfully applied in the characterization of the genetic defect in xeroderma pigmentosum $(26,27)$.

Finally, some therapeutic implications should be considered. Provided the lack of plasmalogens is responsible for radical induced cell damage in disorders like ZS and RCDP, substitution of plasmalogens could prevent further damage. Wilson et al. (28) administered ether lipids orally in ZS and achieved a partial normalization of red blood cell plasmalogen levels. Supplementing RCDP patients with plasmalogens could be even more promising in that only plasmalogen synthesis is deficient in those patients.

\section{Acknowledgments}

The authors wish to thank Prof. Dr. F. Paltauf, Technical University of Graz for his generous gift of 1-O-hexadecyl-sn-glycerol, Dr. P. Wolf, Department of Dermatology, University of Graz for measuring the UV intensity, and M. Czapka for tissue culture and expert technical assistance.

This work was supported by the Fonds zur Förderung der wissenschaftlichen Forschung, projects P7216-MED and P7848-MED, and National Institutes of Health grants RR-00052 and HD-24061.

\section{References}

1. Opitz, J. 1985. The Zellweger syndrome: book review and bibliography. Am. J. Med. Genet. 22:419-426.

2. Lazarow, P., and H. W. Moser. 1989. Disorders of peroxisome biogenesis. In The Metabolic Basis of Inherited Disease. 6th edition. C. R. Scriver, A. L. Beaudet, W. S. Sly, and D. Valle, editors. McGraw Hill Inc., New York. 14791509.

3. Heymans, H. S. A., J. W. E. Oorthuys, G. Nelck, R. J. A. Wanders, and R. B. H. Schutgens. 1985. Rhizomelic chondrodysplasia punctata: another peroxisomal disorder. N. Engl. J. Med. 313:187-188.

4. Hoefler, G., S. Hoefler, P. A. Watkins, W. W. Chen, A. Moser, V. Baldwin, B. McGillivary, J. Charrow, J. M. Friedman, L. Rutledge, et al. 1988. Biochemical abnormalities in rhizomelic chondrodysplasia punctata. J. Pediatrics. 112:726-733.

5. Schram, A. W., S. Goldfischer, C. W. T. van Roermund, E. M. BrouwerKelder, J. Collins, T. Hashimoto, H. S. A. Heymans, H. van den Bosch, R. B. H. Schutgens, J. M. Tager, et al. 1987. Human peroxisomal 3-oxoacyl-coenzyme A thiolase deficiency. Proc. Natl. Acad. Sci. USA. 84:2494-2496.
6. Watkins, P. A., W. W. Chen, C. J. Harris, G. Hoefler, S. Hoefler, D. C. Blake, Jr., A. Balfe, R. I. Kelley, A. B. Moser, M. E. Beard, et al. 1989. Peroxisomal bifunctional enzyme deficiency. J. Clin. Invest. 83:771-777.

7. Zoeller, R. A., O. H. Morand, and C. R. H. Raetz. 1988. A possible role for plasmalogens in protecting animal cells against photosensitized killing. J. Biol. Chem. 263:11590-11596.

8. Zoeller, R. A., L. A. Allen, M. J. Santos, P. B. Lazarow, T. Hashimoto, A. M. Tartakoff, and C. R. H. Raetz. 1989. Chinese hamster ovary cell mutants defective in peroxisome biogenesis: comparison to Zellweger syndrome. J. Biol. Chem. 264:21872-21878.

9. Slater, T. F. 1984. Free-radical mechanisms in tissue injury. Biochem. J. 222:1-15.

10. Moser, H. W., A. B. Moser, N. Kawamura, J. Murphy, K. Suzuki, H. Schaumburg, Y. Kishimoto, and A. Milunsky. 1980. Adrenoleukodystrophy: elevated C26 Fatty Acid in cultured skin fibroblasts. Ann. Neurol. 7:542-549.

11. Poulos, A., P. Sharp, A. J. Fellenberg, and D. M. Danks. 1985. Cerebrohepato-renal (Zellweger) syndrome, adrenoleukodystrophy, and Refsum's disease: plasma changes and skin fibroblast oxidase. Hum. Genet. 70:172-177.

12. Roscher, A., B. Molzer, H. Bernheimer, S. Stöckler, I. Mutz, and F. Paltauf. 1985. The cerebro-hepato-renal (Zellweger) syndrome. An improved method for the biochemical diagnosis and its potential for prenatal diagnosis. Pediatr. Res. 19:930-933.

13. Bjorkhem, I., and O. Falk. 1983. Assay of the major bile acids in serum by isotope dilution-mass spectrometry. Scand. J. Clin. Lab. Invest. 43:163-170.

14. Van den Berg, C. A., H. Breukelman, H. Elzinga, J. M. F. Trijbels, L. A. H. Monnens, and F. A. J. Muskiet. 1986. Determination of pipecolic acid in urine and plasma by isotope dilution mass fragmentography. Clin. Chim. Acta. 159:229-237.

15. Lazarow, P. W., Y. Fujiki, G. M. Small, P. A. Watkins, and H. W. Moser. 1986. Presence of the peroxisomal 22-kDa integral membrane protein in the liver of a person lacking recognizable peroxisomes (Zellweger syndrome). Proc. Natl. Acad. Sci. USA. 87:9193-9197.

16. Roscher, A. A., S. Hoefler, G. Hoefler, E. Paschke, F. Paltauf, A. B. Moser, and H. W. Moser. 1989. Genetic and phenotypic heterogeneity in disorders of peroxisome biogenesis: a complementation study involving cell lines from 19 patients. Pediatr. Res. 26:67-72.

17. Naidu, S., G. Hoefler, P. A. Watkins, W. W. Chen, A. B. Moser, S Hoefler, N. Rance, J. M. Powers, M. Beard, W. R. Green, et al. 1988. Neonatal seizures and retardation in a female with biochemical features of X-linked ALD: a possible new peroxisomal disease entity. Neurology. 38:1100-1107.

18. Bligh, E. G., and W. J. Dyer. 1959. A rapid method of total lipid extraction and purification. Can. J. Biochem. Physiol. 37:911-917.

19. Lowry, O. H., N. J. Rosebrough, A. L. Farr, and R. J. Randall. 1951 Protein measurement with the Folin phenol reagent. J. Biol. Chem. 193:265-275.

20. Hermetter, A., B. Rainer, E. Ivessa, E. Kalb, J. Loidl, A. Roscher, and F. Paltauf. 1989. Influence of plasmalogen deficiency on membrane fluidity of human skin fibroblasts: a fluorescence anisotropy study. Biochim. Biophys. Acta. 978:151-157.

21. Gatt, S., J. Bremer, and H. Osmundsen. 1988. Pyrene dodecanoic acid coenzyme A ester: peroxisomal oxidation and chain shortening. Biochim. Biophys. Acta. 958:130-133.

22. Wanders, R. J. A., C. W. T. van Roermund, M. J. A. van Wijland, R. B. H. Schutgens, J. Heikoop, H. van den Bosch, A. W. Schram, and J. M. Tager. 1987 Peroxisomal fatty acid beta-oxidation in relation to the accumulation of very long chain fatty acids in cultured skin fibroblasts from patients with Zellweger syndrome and other peroxisomal disorders. J. Clin. Invest. 80:1778-1183.

23. Lazo, O., M. Contereas, A. Bushan, W. Stanley, and I. Singh. 1989. Adrenoleukodystrophy: impaired oxidation of fatty acids due to peroxisomal lignoceroyl-CoA ligase deficiency. Arch. Biochem. Biophys. 270:722-728.

24. Brul, S., A. Westerveld, A. Strijland, R. J. A. Wanders, A. W. Schram, H. S. A. Heymans, R. B. H. Schutgens, H. van den Bosch, and J. M. Tager. 1988. Genetic heterogeneity in the cerebro-hepato-renal (Zellweger) syndrome and other inherited disorders with a generalized impairment of peroxisomal functions: a study using complementation analysis. J. Clin. Invest. 81:1710-1715.

25. McGuinness, M. C., A. B. Moser, H. W. Moser, and P. A. Watkins. 1990. Peroxisomal disorders: complementation analysis using $\beta$-oxidation of very long chain fatty acids. Biochem. Biophys. Res. Commun. 172:364-369.

26. Teitz, T., T. Naiman, S. S. Avissar, S. Bar, H. Okayama, and D. Canaani. 1987. Complementation of the UV-sensitive phenotype of a xeroderma pigmentosum human cell line by transfection with a cDNA clone library. Proc. Natl. Acad. Sci. USA. 84:8801-8804.

27. Tanaka, K., N. Miura, I. Satokata, I. Miyamoto, M. C. Yoshida, Y. Satoh, S. Kondo, A. Yasui, H. Okayama, and Y. Okada. 1990. Analysis of a human DNA excision repair gene involved in group A xeroderma pigmentosum and containing a zinc-finger domain. Nature (Lond.). 348:73-76.

28. Wilson, G. N., R. G. Holmes, J. Custer, J. I. Lipkowitz, J. Stover, N. Datta, and A. Hajra. 1986. Zellweger syndrome: diagnostic assays, syndrome delineation, and potential therapy. Am. J. Med. Genet. 24:69-82. 